\title{
A Theoretical Characterization of Time Dependent Materials by Using a Hyperlogistic-Type Model
}

\author{
Marc Delphin Monsia ${ }^{1} \&$ Yélomè Judicaël Fernando Kpomahou ${ }^{2}$ \\ ${ }^{1}$ Département de Physique, Université d'Abomey-Calavi, Abomey-Calavi, Cotonou, Bénin \\ ${ }^{2}$ Doctorant au Département de Physique, Université d'Abomey-Calavi, Abomey-Calavi, Bénin \\ Correspondence: Marc Delphin Monsia, Département de Physique, Université d'Abomey-Calavi, \\ Abomey-Calavi, 09 B. P. 305, Cotonou, Bénin. Tel: 229-95-568-187. E-mail: monsiadelphin@yahoo.fr
}

Received: January 9, 2012

Accepted: February 2, $2012 \quad$ Online Published: May 24, 2012

doi:10.5539/mer.v2n1p36

URL: http://dx.doi.org/10.5539/mer.v2n1p36

\begin{abstract}
In this work, the classical mechanical Voigt model is modified and extended to finite deformations by using a rational elastic spring force function to describe accurately the nonlinear time-dependent deformation response of some viscoelastic materials. As theoretical results, a hyperlogistic-type function has been found as the deformation versus time relationship. This growth model appeared powerful to reproduce mathematically as shown by numerical works, any S-shaped experimental data. Compared with some previous models, the present one-dimensional formulation gives the advantage to assure or to control via an explicit material parameter, to speak, via the coefficient of inertia, the nonlinearity of the model. The proposed model demonstrated then the importance to consider in the material modeling the inertial coefficient.
\end{abstract}

Keywords: rational elastic spring force function, hyperlogistic-type function, Lambert-type equation, viscoelasticity, Voigt model

\section{Introduction}

In mechanics and, engineering and biomedical applications, analytical viscoelastic models are required for the prediction and simulation of time dependent behavior of materials. In viscoelastic modeling, two approaches are used to estimate the material response to mechanical excitation: linear and nonlinear theories. But, the well-known established linear viscoelastic theory is only valid in the low deformations or stresses domain. So that, when materials undergo large deformations, nonlinear theories are needed for describing their rheological properties. Viscoelastic materials are time and history-dependent properties materials. Thus, their response is characterized by elastic, viscous and inertial contributions. These contributions are in general highly nonlinear. In this way, the constitutive equations of viscoelastic materials are nonlinear and must mathematically relate the stress, strain and their higher time derivatives (Bauer et al., 1979; Bauer, 1984). Constitutive equations are often determined from combinations of springs and dashpots arranged in series and/or parallel (Alfrey \& Doty, 1945) or from the Boltzmann integral equation. However, these classical Maxwell or Voigt models or also their complex combinations like that the Boltzmann single integral equation cannot describe accurately the nonlinear material properties. Then, it becomes necessary to modify the classical linear models for including nonlinear terms in order to take into account nonlinear properties of viscoelastic materials. Many predictive models are shown to be based on the extension of linear rheological models to finite deformations (Corr et al., 2001; Monsia 2011a, 2011b, 2011c, 2011d, 2011e). The modifications consist often to incorporate nonlinear elastic springs and/or nonlinear dashpots in the classical linear models. Another method considers that the material functions depend on the magnitude of the stress, strain or strain rate. The resulting model is interesting since, according to Alfrey and Doty (1945), it estimates the material properties in terms of differential equations which can be solved for a large variety of transient conditions. However, these nonlinear formulations lead often to solve nonlinear differential equations which are generally non-integrable. Therefore, nonlinear rheological theories are not a simple task and appear more difficult to formulate than linear viscoelastic models. Moreover, in viscoelasticity theory, there are only a few theoretical models expressed with constant-value material coefficients (Corr et al., 2001). Thus, constant coefficient nonlinear viscoelastic models are needed. In this regard, Corr et al. (2001) derived a Riccati differential equation that is useful to predict the time dependent stress induced in viscoelastic materials by extending the Maxwell fluid model to finite deformations. Monsia (2011b) using a 
second-order elastic spring in series with a classical Voigt element, which is an extended form of the standard linear solid to finite strains, constructed a hyperlogistic-type equation to reproduce the nonlinear time-dependent stress response of some viscoelastic materials. Monsia (2011c) again, developed a single differential constitutive equation derived from a nonlinear standard solid model made of a polynomial elastic spring combined with a classical Voigt element in series for the prediction of time-dependent nonlinear stress of a class of viscoelastic materials. In (Monsia, 2011d), the author formulated a nonlinear four-parameter rheological Voigt model made of a nonlinear Voigt element arranged in series with a classical linear Voigt element with constant material coefficients for representing the nonlinear stiffening response of the initial low-load portion and the softening, that is to say, the S-shaped mechanical behavior of some viscoelastic materials. Recently, Monsia (2011e) developed a simple nonlinear generalized Maxwell fluid model composed of nonlinear spring connected with a nonlinear dashpot in series obeying a power-law with constant material coefficients for representing accurately the time-dependent behavior of some viscoelastic materials. However, in many viscoelastic models, such as the models mentioned previously, in exception of the model (Monsia, 2011a), the inertia of the system under study is neglected. Moreover, both nonlinear elastic and viscous contributions are also not taken in general into consideration simultaneously, for mathematical simplification reasons of constitutive equations. To overcome these above mentioned difficulties, Bauer (1984) by studying the arterial wall rheological properties, developed a theory derived from the classical Voigt model, which consists to decompose the total stress acting on the material as the sum of three components, that is, the elastic, viscous and inertial stresses. The first of which is a power series of strain, the second is a first time derivative of a similar power series of strain, and the third is a second time derivative of a similar power series of strain. This hypothesis, consisting to express the stress as a sum of three elementary stresses, has been after used by many authors (Armentano et al., 1995; Gamero et al., 2001; Monsia et al., 2009) for the complete characterization of arterial behavior. In (Monsia et al., 2009), following the Bauer's approach (1984), the elastic, viscous and inertial stresses are expanded in power series of strain. Recently, Monsia (2011a) using also the Bauer's method (1984) and expressing the elastic stress as an asymptotic expansions in powers of deformation, the viscous stress as a first time derivative of a similar asymptotic expansions in powers of deformation, and the inertial stress as a second time derivative of a similar asymptotic expansions in powers of deformation, developed a hyperlogistic equation that represents successfully the dynamical response of a variety of materials. Recently again, Monsia (2011f) using again the Bauer's approach (1984), proposed a nonlinear rheological model based on a substitution of linear elastic and damping forces in Voigt model by nonlinear elastic and damping forces with inclusion of a body for providing a theoretical basis to empirical exponential or logistic formulas used by several authors for fitting the stress-strain experimental data of arteries. Very lately, Monsia (2011g) by application of the Bauer's theory (1984), developed successfully for a variety of materials a nonlinear rheological model that can be used to predict and describe their dynamical behavior. By using a hyperbolic law as elastic spring force function (Monsia, 2011g), the strain versus time variation has been described as a hyper-exponential type function that appeared useful to reproduce the typical nonlinear exponential deformation of some viscoelastic materials. By considering also the same hyperbolic law and applying the Bauer's theory, Monsia (2012) formulated for some materials, a rheological model to describe successfully their time dependent response under an applied constant load. The hyper-exponential type or hyperlogistic type function obtained as theoretical results were powerful to describe the nonlinear creep curves of the mechanical system studied.

In this work, in order to describe successfully the time-dependent deformation behavior of some viscoelastic materials, a one-dimensional nonlinear particular Voigt model developed by using the Bauer's theory (1984) with constant material coefficients, is proposed. In this model, the elastic spring force function is expressed as a rational function of deformation. The obtained governing equation, to speak, the Lambert-type differential equation gives, by using suitable boundary conditions, the time-dependent deformation in the viscoelastic material studied as a hyperlogistic-type function. Numerical works are carried out to illustrate the sensitivity of the model to material parameters.

\section{Formulation of the Mechanical Model}

\subsection{Theoretical Considerations}

The theoretical model will be described in this section and the governing equation including the nonlinear elastic force, damping and inertia forces will be also derived. Since viscoelastic materials exhibit elastic, viscous and inertial nonlinearities, their mechanical responses are nonlinear time-dependent. Then, their characterization needs consistent mathematical models. For this, the use of nonlinear viscoelasticity is suggested (Bauer et al., 1979; Bauer, 1984). To construct our proposed rheological model, we consider the Bauer's theory (1984) as formulated previously in (Monsia, 2011g, 2012). This theory (Bauer, 1984) brings important modifications to the 
Voigt model for overcoming the preceding mentioned difficulties in viscoelastic modeling. In the Bauer's theory (1984), the total exciting stress is divided into three components: the pure elastic stress, the pure viscous stress and the pure inertial stress. The viscous and inertial stresses are derived as first and second time derivatives of a similar function to the pure elastic stress. Therefore, the superposition of these three stress components, for a nonlinear elastic spring force function $\varphi(\varepsilon)$ of deformation $\varepsilon$, which is a scalar function, leads to the fundamental equation (Monsia, 2011g; Monsia, 2012)

$$
\ddot{\varepsilon} \frac{d \varphi}{d \varepsilon}+\dot{\varepsilon}^{2} \frac{d^{2} \varphi}{d \varepsilon^{2}}+\frac{b}{c} \dot{\varepsilon} \frac{d \varphi}{d \varepsilon}+\frac{a}{c} \varphi(\varepsilon)=\frac{1}{c} \sigma_{t}
$$

where the dot over a symbol denotes a differentiation with respect to time and the inertial coefficient $c$ is different from zero. The coefficients $a$ and $b$ are respectively the stiffness and viscosity modules. These all three coefficients are time independent material parameters. $\sigma_{t}$, which is a scalar function, represents the total exciting stress acting on the material studied. To go further, it is needed to specify the nonlinear elastic force function $\varphi(\varepsilon)$. According to the Bauer's theory (1984), the nonlinear elastic spring force function $\varphi(\varepsilon)$, for small deformations, must behave linearly, to speak, must obey to the Hooke's law. Based on this criterion, for the present one-dimensional mechanical model, $\varphi(\varepsilon)$ can empirically be chosen as the explicit rational function

$$
\varphi(\varepsilon)=\frac{1}{1-\varepsilon}
$$

For small strain $(\varepsilon<<1)$, Equation (2) behaves as a linear hookean spring function. The nonlinear elastic force $\varphi(\varepsilon)$ stiffens as $\varepsilon \rightarrow 1$ and has a vertical asymptote at $\varepsilon=1$. By, using equation (2), Equation (1) becomes

$$
\sigma_{t}=\frac{a}{1-\varepsilon}+\frac{b \dot{\varepsilon}}{(1-\varepsilon)^{2}}+\frac{c \ddot{\varepsilon}(1-\varepsilon)+2 c \dot{\varepsilon}^{2}}{(1-\varepsilon)^{3}}
$$

Equation (3) represents mathematically in the single differential form the relation between the total exciting stress $\sigma_{t}$ and the resulting strain $\varepsilon$. This equation is a second-order nonlinear ordinary differential equation in $\varepsilon$ for a chosen exciting stress $\sigma_{t}$.

\subsection{Dimensionalization}

The deformation $\varepsilon(t)$ is a dimensionless quantity. Then in Eq. (3) the coefficients have the following dimensions. Let $M, L$ and $T$ denote the mass, length and time dimension respectively, the dimension of the stress varies as $M L^{-1} T^{-2}$. Therefore, the dimension of $a$ varies as $M L^{-1} T^{-2}$, that of $b$ varies as $M L^{-1} T^{-1}$, and that of $c$ varies as $M L^{-1}$ (mass per unit length).

2.3 Solution Using a Stress $\sigma_{t}=0$

\subsubsection{Evolution Equation of the Deformation $\varepsilon(t)$}

In the absence of exciting stress $\left(\sigma_{t}=0\right)$, the internal dynamics of the viscoelastic material under study is expressed by the following governing evolution Lambert-type equation

$$
\frac{a}{1-\varepsilon}+\frac{b \dot{\varepsilon}}{(1-\varepsilon)^{2}}+\frac{c \ddot{\varepsilon}(1-\varepsilon)+2 c \dot{\varepsilon}^{2}}{(1-\varepsilon)^{3}}=0
$$

which can be also written in the form

$$
c \ddot{\varepsilon}+2 c \frac{\dot{\varepsilon}^{2}}{1-\varepsilon}+b \dot{\varepsilon}+a(1-\varepsilon)=0
$$

or

$$
\ddot{\varepsilon}+2 \frac{\dot{\varepsilon}^{2}}{1-\varepsilon}+\lambda \dot{\varepsilon}+\omega_{o}^{2}(1-\varepsilon)=0
$$

where $\lambda=\frac{b}{c}, \omega_{o}^{2}=\frac{a}{c}$

For the inertial coefficient $c=0$, Equation (6) becomes a first-order linear ordinary differential equation. The 
inertial coefficient $c$ that must be, here, different from zero, assures then the material nonlinear dynamics.

\subsubsection{Solving Time-deformation Equation}

In order to solve Equation (6) we proceed to the following change of variable

$$
x=1-\varepsilon
$$

Thus, Equation (6) becomes

$$
\ddot{x}-2 \frac{\dot{x}^{2}}{x}+\lambda \dot{x}-\omega_{o}^{2} x=0
$$

In (Monsia, 2011a, 2011g; Monsia, 2012), a similar equation to (8) is already solved. Thus, the use of the transformation

$$
f=\frac{\dot{x}}{x}
$$

allows the differential equation (8) to be solvable in closed-form solution. Substituting this change of variable into Equation (8), we obtain after a few algebraic manipulations

$$
\dot{f}=f^{2}-\lambda f+\omega_{o}^{2}
$$

Equation (10) is a first-order nonlinear Riccati differential equation for the variable $f$ which has the strain rate dimension. Using suitable boundary conditions, that is to say

$$
t \rightarrow 0, \lim f(t)=f_{o},
$$

and

$$
t \rightarrow+\infty, \lim f(t)=0
$$

one can obtain by integration the following analytical solution

$$
x(t)=x_{\max }[1+q \exp (-\lambda \delta t)]^{-\frac{1}{n}}
$$

in which $\delta=\sqrt{1-4 \frac{\omega_{o}^{2}}{\lambda^{2}}}, f_{2}=\frac{\lambda}{2}(1-\delta), q=\frac{f_{o}}{f_{2}-f_{o}}, n=\frac{\lambda \delta}{f_{2}}$, and the condition $t \rightarrow+\infty, \lim x(t)=x_{\max }=K$.

By considering now Equation (7) one can easily obtain the desired strain versus time variation

$$
\varepsilon(t)=1-K[1+q \exp (-\lambda \delta t)]^{-\frac{1}{n}}
$$

Equation (12) gives the time variation of the strain in the viscoelastic material under consideration. It represents the strain versus time relationship as a hyperlogistic-type model that is powerful to reproduce any S-shaped curve.

\section{Numerical Results and Discussion}

In this part, using an own computer program, some numerical examples are presented to demonstrate the predictive ability of the model to reproduce the mechanical response of the material studied. The dependence of the strain versus time curve on the material parameters is also discussed. In the following of this work the numerical illustrations are investigated at the fixed value $K=-1$. 


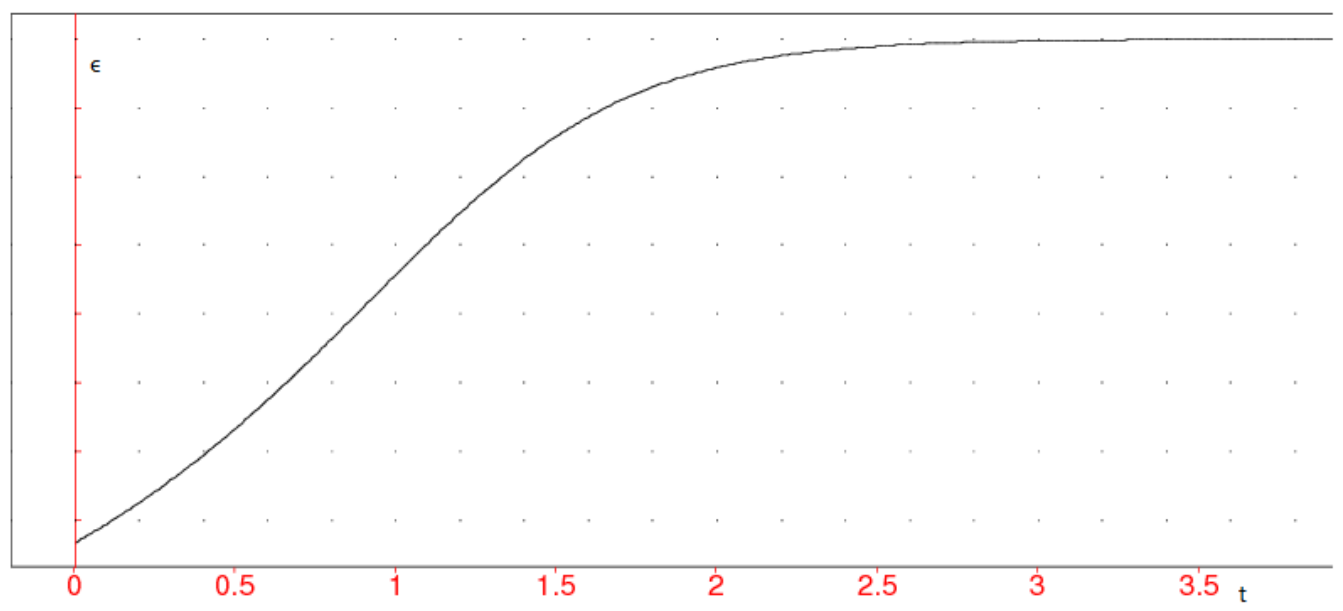

Figure 1. Typical strain versus time curve showing an asymptotical value

Figure 1 shows the typical strain versus time curve with an increase until a peek asymptotical value, obtained from Equation (12) with the fixed value of coefficients at $\lambda=4.9275, \omega_{o}=2, f_{o}=1$. It may be observed from Figure 1 the capability of the model to represent mathematically and accurately the typical exponential deformation of a variety of viscoelastic materials, for example, soft biological materials as shown in (Monsia 2011g). The time-dependent strain curve is nonlinear, with a nonlinear beginning initial portion, and illustrates then the sigmoid deformation behavior of the viscoelastic material studied. Figure 2, 3 and 4 illustrates the effect of material parameters on the strain. The effects of these parameters are investigated by varying step by step one coefficient while keeping the other two constant.

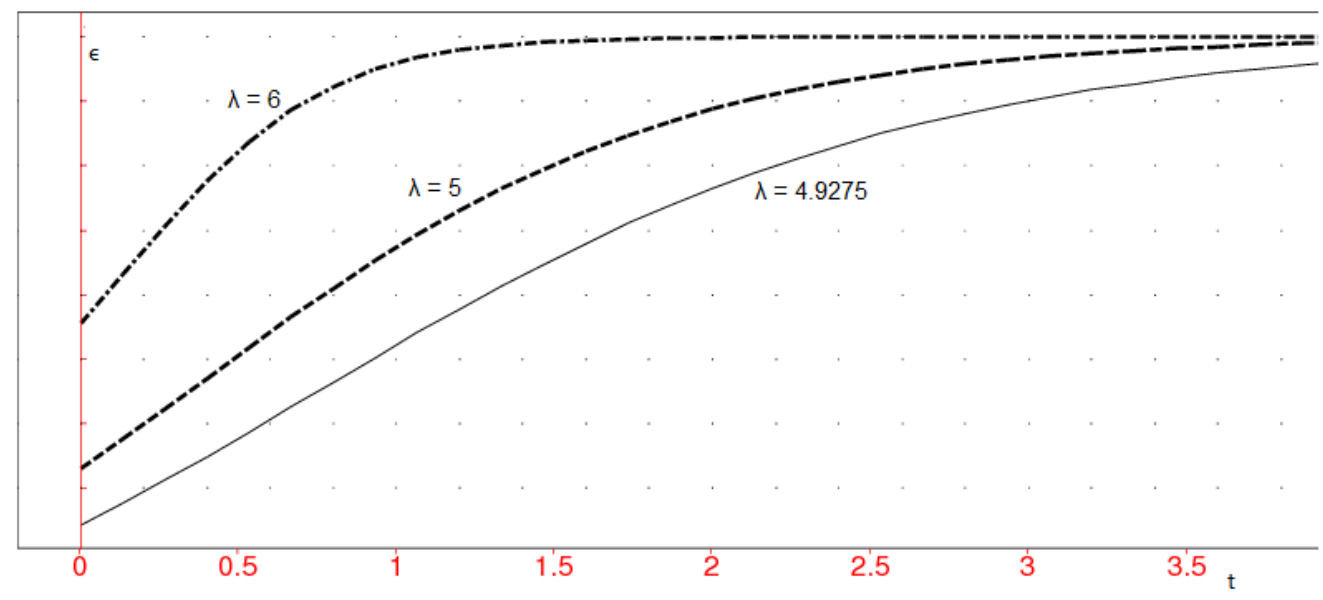

Figure 2. Strain-time curves at various values of the coefficient $\lambda$

Figure 2 exhibits the dependence of the strain versus time curve on the linear viscous damping term $\lambda$. The graph indicates that an increase $\lambda$, increases the initial value of the strain, and shortens the time needed to attain the maximum strain. The solid line corresponds to $\lambda=4.9275$, the dashed to $\lambda=5$, and the dash-dot line to $\lambda=6$. The other parameters are $\omega_{o}=2.41397, f_{o}=1$. 


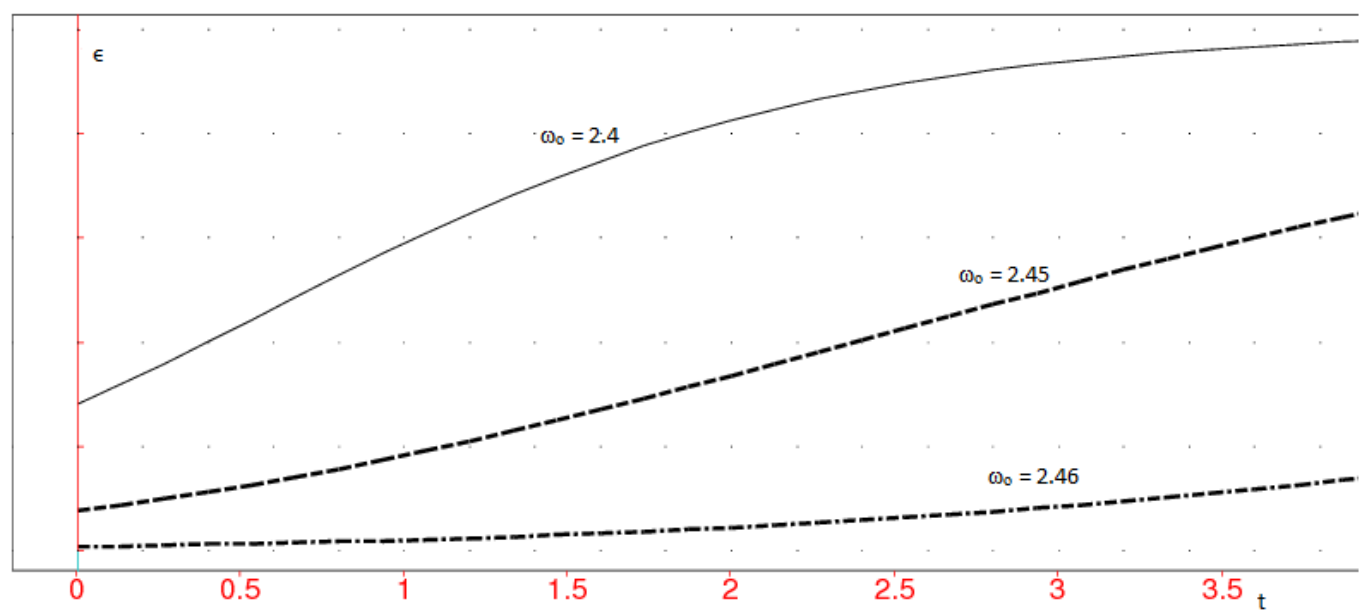

Figure 3. Strain-time curves showing the effect of the natural frequency $\omega_{o}$

We observe from Figure 3 that an increasing natural frequency $\omega_{o}$, decreases the initial value of the strain, and increases the time required to reach the maximum strain. The solid line corresponds to $\omega_{o}=2.4$, the dashed line to $\omega_{o}=2.45$, and the dash-dot line to $\omega_{o}=2.46$. The other parameters are $\lambda=4.9275, f_{o}=1$.

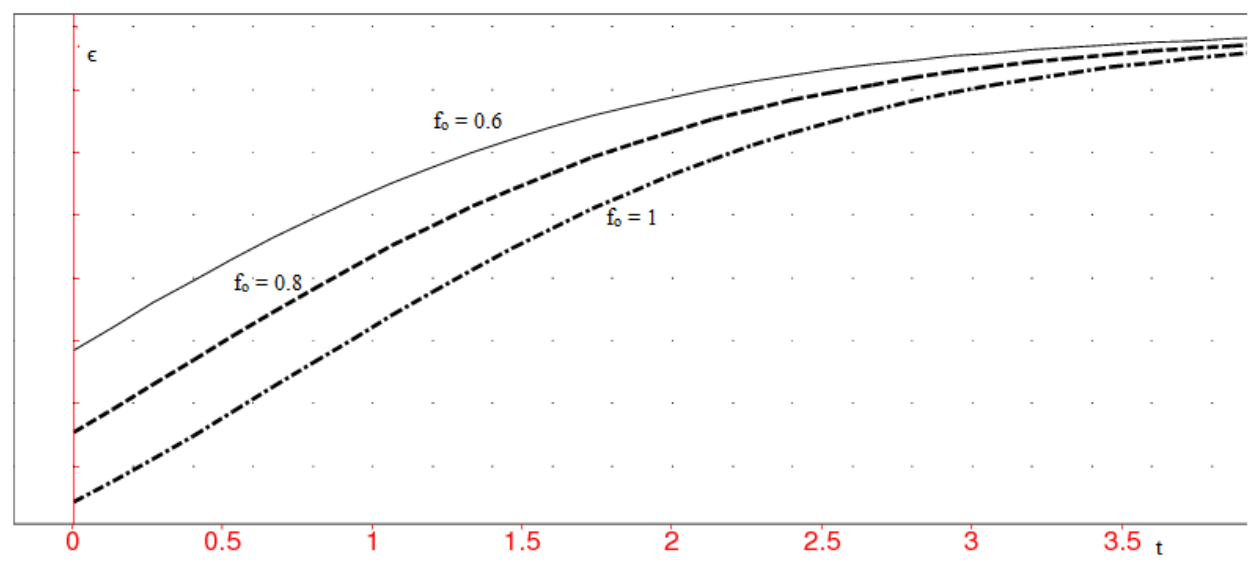

Figure 4. Strain versus time curves at three different values of $f_{o}$

The strain curves at various values of the relative initial strain rate $f_{o}$ for the material under study are shown in Figure 4. An increasing $f_{o}$, decreases the initial strain value and increases the time necessary to attain the peak strain. The solid line corresponds to $f_{o}=0.6$, the dashed to $f_{o}=0.8$, and the dash-dot line to $f_{o}=1$. The other parameters are $\lambda=4.9275, \omega_{o}=2.41397$.

For the present model, the function $\varphi(\varepsilon)$ is given by a rational function. The choice of this law for describing the elastic spring force function is inspired by the nonlinear elastic FENE spring model (Herrchen \& Öttinger, 1997). This proposed law is also justified by the fact that for $0<\varepsilon<1, \varphi(\varepsilon)$ reduces to a geometric series formula so that, for small deformations, $\varphi(\varepsilon)$ shows linear behavior as expected. In this regard, the rational law considered for capturing the pure nonlinear elastic behavior of the material studied, agrees very well with the polynomial function of deformation used by Bauer (1984). Another feature which involves the choice of this rational law is that $\varphi(\varepsilon)$ becomes infinite force for a finite deformation tending towards unity $(\varepsilon \rightarrow 1)$, that is to say, for a strain range of about $100 \%$. For $\varepsilon>>1$ (to say $\varepsilon \rightarrow+\infty$ ), $\varphi(\varepsilon)$ tends towards a finite value zero. The present study, compared with the preceding models (Monsia 2011g; Monsia 2012), provides the advantage to assure or to control the nonlinearity of the model via an explicit material parameter, that is to say, via the coefficient of inertia $c$. Thus, the present model revealed that the mass of materials contributes highly to 
nonlinear properties of these mechanical systems. By numerical examples, we illustrated the predictive capability of the proposed model. There exist in mechanics literature a number of rheological models that describe more or less accurately the deformation behavior of viscoelastic materials. A few models, however, take into consideration in their description the inertial contribution, and moreover, simultaneously the nonlinearities due to the inertial, viscous and elastic effects. As viscoelastic materials are very characterized by nonlinear mechanical properties, the linear viscoelastic theory becomes inapt to represent the material response. To overcome this fact, in this study, we proposed a one-dimensional nonlinear rheological model on the basis of the Voigt model including simultaneously the nonlinear inertial, viscous and elastic contributions of the material considered by applying the Bauer's theory (Bauer 1984) that is very well suited for a complete characterization of viscoelastic materials. The present model is constructed from the hypothesis following which the elastic spring force can be articulated as a rational law of deformation and the viscous and inertial stresses as the first and second time derivatives of similar rational function of deformation. This approach led to obtain the present three material constant parameters model of curve. The resulting strain versus time relationship is expressed as a hyperlogistic-type function. It is well known that hyperlogistic-type function is powerful to represent mathematically any S-shaped curve. Thus, the proposed model is well indicated, as shown by numerical illustrations, to reproduce correctly the deformation response noted in some viscoelastic materials undergoing large deformations.

\section{Conclusions}

We presented in this paper for a variety of materials a nonlinear rheological model by using a rational elastic spring force in order to predict their finite deformation behavior. More precisely, their elastic, viscous and inertial nonlinearities are simultaneously taken into consideration. Numerical examples are performed to illustrate the predictive capability of the proposed model. The model appeared to be applicable to describe the nonlinear sigmoid deformation versus time curve of a variety of viscoelastic materials. It is worth mentioning that the model allows study of the time-dependent stress behavior as well as the stress versus strain curve, but this study will be done as subsequent work.

\section{References}

Alfrey, T., \& Doty, P. (1945). The Methods of Specifying the Properties of Viscoelastic Materials. Journal of Applied Physics, 16, 700-713. http://dx.doi.org/10.1063/1.1707524

Armentano, R. L., Barra, J. G., Levenson, J., Simon, A., \& Pichel, R. H. (1995). Arterial wall mechanics in conscious dogs. Assessment of viscous, inertial, and elastic moduli to characterize aortic wall behaviour. Circ Res., 76, 468-478.

Bauer, R. D., Busse, R., Schabert, A., Summa, Y., \& Wetterer, E. (1979). Separate determination of the pulsatile elastic and viscous forces developed in the arterial wall in vivo. Pflügers. Arch., 380, 221-226. http://dx.doi.org/10.1007/BF00582900

Bauer, R. D. (1984). Rheological approaches of arteries. Biorheology. Suppl. I, 159-167

Corr, D. T., Starr, M. J., Vanderby, Jr R., \& Best, T. M. (2001). A Nonlinear Generalized Maxwell Fluid Model for Viscoelastic Materials. Journal of Applied Mechanics, 68, 787-790. http://dx.doi.org/10.1115/1.1388615

Gamero, L. G., Armentano, R. L., Barra, J. G., Simon, A., \& Levenson, J. (2001). Identification of Arterial Wall Dynamics in Conscious Dogs. Exp. Physiol., 86, 519-528. http://dx.doi.org/10.1113/eph8602172

Herrchen, M., \& Öttinger, H. C. (1997). A Detailed Comparison of Various FENE Dumbbell Models. Journal of Non-Newtonian Fluid Mechanics. 68, 17-42. http://dx.doi.org/10.1016/S0377-0257(96)01498-X

Monsia, M. D. (2011a). Lambert and Hyperlogistic Equations Models for Viscoelastic Materials: Time-dependent Analysis. Int. J. Mech. Eng., 4, 5-10.

Monsia, M. D. (2011b). A Hyperlogistic-type Model for the Prediction of Time-dependent Nonlinear Behavior of Viscoelastic Materials. Int. J. Mech. Eng., 4, 1-4.

Monsia, M. D. (2011c). A Nonlinear Generalized Standard Solid Model for Viscoelastic Materials. Int. J. Mech. Eng., 4, 11-15.

Monsia, M. D. (2011d). A Modified Voigt Model for Nonlinear Viscoelastic Materials. Int. J. Mech. Eng., 4, $17-20$.

Monsia, M. D. (2011e). A Simplified Nonlinear Generalized Maxwell Model for Predicting the Time Dependent Behavior of Viscoelastic Materials. World Journal of Mechanics., 1, 158-167. 
http://dx.doi.org/10.4236/wjm.2011.13021

Monsia, M. D., Massou, S., \& Moussiliou, S. (2009). Nonlinear Mathematical Model for Viscoelastic Behavior of Arterial Wall. Rev. CAMES-Série A, 08, 12-17.

Monsia, M. D. (2011f). A Riccati Pressure-Length Equation for Arterial Walls Using a Nonlinear Voigt Model. International Journal of Computational Intelligence and Bioinformatics, 3, 71-82.

Monsia, M. D. (2011g). Modeling the Nonlinear Rheological Behavior of Materials with a Hyper-Exponential Type Function. Mechanical Engineering Research, 1, 103-109. http://dx.doi.org/10.5539/mer.v1n1p103

Monsia, M. D. (2012). A Nonlinear Mechanical Model for Predicting the Dynamics Response of Materials under a Constant Loading. Journal of Materials Science Research, 1, 90-100. http://dx.doi.org/10.5539/jmsr.v1n1p90 